Published in final edited form as:

Otolaryngol Head Neck Surg. 2018 April ; 158(4): 736-744. doi:10.1177/0194599818758247.

\title{
Hearing trajectory in children with congenital cytomegalovirus infection
}

\author{
Tatiana M. Lanzieri, M.D. ${ }^{1}$, Winnie Chung, Au.D ${ }^{2}$, Jessica Leung, M.P. ${ }^{1}$, A. Chantal \\ Caviness, M.D. ${ }^{3}$, Jason L. Baumgardner, M.S.P.H. ${ }^{1}$, Peggy Blum, Au.D ${ }^{4}$, Stephanie R. \\ Bialek, M.D. ${ }^{1}$, Gail Demmler-Harrison, M.D. ${ }^{3,4}$, and for the Congenital Cytomegalovirus \\ Longitudinal Study Group
}

${ }^{1}$ National Center for Immunization and Respiratory Diseases, Centers for Disease Control and Prevention, Atlanta, GA, USA

${ }^{2}$ National Center on Birth Defects and Developmental Disabilities, Centers for Disease Control and Prevention, Atlanta, GA, USA

${ }^{3}$ Baylor College of Medicine, Houston, TX, USA

${ }^{4}$ Texas Children's Hospital, Houston, TX, USA

\section{Abstract}

Objectives-Compare hearing trajectories among children with symptomatic and asymptomatic congenital CMV infection through age 18 years and identify brain abnormalities associated with sensorineural hearing loss (SNHL) in asymptomatic case-patients.

Study Design—Longitudinal prospective cohort study.

Setting-Tertiary medical center.

Subjects and Methods-Ninety-six (4 symptomatic and 92 asymptomatic) case-patients identified through hospital-based newborn CMV screening during 1982-1992 and 72 symptomatic case-patients identified through referrals during 1993-2005. We used growth curve modeling to analyze hearing thresholds $(0.5-8 \mathrm{kHz})$ by ear with increasing age and Cox regression to determine abnormal findings on head computed tomography (CT) scan associated with SNHL (hearing threshold $\geq 25 \mathrm{~dB}$ in any audiometric frequency) among asymptomatic case-patients.

Results-Fifty-six (74\%) symptomatic and 20 (22\%) asymptomatic case-patients had SNHL: congenital/early-onset SNHL was diagnosed in $78(51 \%)$ and $10(5 \%)$ ears, respectively, and delayed-onset SNHL in $25(17 \%)$ and 20 (11\%) ears; 49 (32\%) and 154 (84\%) ears had normal hearing. In affected ears, all frequency-specific hearing thresholds worsened with age. Congenital/ early-onset SNHL was significantly worse (severe-profound range [>70dB]) than delayed-onset

\footnotetext{
Address correspondence to: Tatiana M. Lanzieri, National Center for Immunization and Respiratory Diseases, Center for Disease Control and Prevention, 1600 Clifton Rd NE, Mail Stop A-34, Atlanta GA, 30333, tmlanzieri@cdc.gov, 404-639-3031.

Financial Disclosure: The authors have no financial relationships relevant to this article to disclose.

Potential Conflict of Interest: The other authors have no potential conflicts to disclose.

Disclaimer: The findings and conclusions in this article are those of the authors and do not necessarily represent the official position of the Centers for Disease Control and Prevention.
} 
SNHL (mild-moderate range [26-55db]). Frequency-specific hearing thresholds were significantly different between symptomatic and asymptomatic case-patients at $0.5-1 \mathrm{kHz}$ but not at higher frequencies $(2-8 \mathrm{kHz})$. Among asymptomatic case-patients, white matter lucency was significantly associated with SNHL by age 5 years (hazard ratio $=4.4 ; 95 \%$ confidence interval: 1.3-15.6).

Conclusion-Congenital/early-onset SNHL frequently resulted in severe to profound loss in both symptomatic and asymptomatic case-patients. White matter lucency in asymptomatic casepatients was significantly associated with SNHL by age 5 years.

\section{Keywords}

congenital cytomegalovirus infection; sensorineural hearing loss

\section{INTRODUCTION}

In the United States, an estimated 15-20\% of all cases of bilateral moderate to profound sensorineural hearing loss (SNHL) among young children are attributable to congenital cytomegalovirus (CMV) infection. ${ }^{1}$ Among an estimated 20,000 (0.5\%) U.S. children born with congenital CMV infection annually ${ }^{2,3}$, approximately $10 \%$ may present with symptomatic disease at birth. ${ }^{4}$ Moderately to severely symptomatic disease manifests with central nervous system involvement (microcephaly, chorioretinitis, SNHL, or brain abnormalities) or multiple signs, such as thrombocytopenia, petechiae, hepatosplenomegaly, hepatitis, and intrauterine growth restriction, attributable to congenital CMV infection. ${ }^{5}$ Mildly symptomatic disease may occur with one or two signs that are mild and transient (e.g. mild hepatomegaly). ${ }^{5}$

Currently, congenital CMV infection with no apparent abnormalities except SNHL is defined as asymptomatic congenital cytomegalovirus infection with isolated SNHL. ${ }^{5}$ The proportion of infants diagnosed with SNHL within 8 weeks of life is much higher among those with symptomatic disease at birth compared to asymptomatic ones (38\% vs. 5\%). However, infants with asymptomatic infection may account for a greater number of SNHL cases. ${ }^{4}$ Moreover, an estimated 5\% of children with asymptomatic congenital CMV infection -- about 900 children annually in the United States -- have severe hearing loss in at least one ear by age 12 months. ${ }^{6}$ Half of these children meet current candidacy criteria for cochlear implantation by age 5 years. ${ }^{6}$

Microcephaly and abnormal head computed tomography (CT) scan findings indicative of tissue destruction and dysplastic growth have been associated with increased risk of SNHL among infants with symptomatic congenital CMV disease at birth. ${ }^{7}$ Among infants with asymptomatic congenital CMV infection, periventricular radiolucency as well as intracranial calcifications in head CT scans have been associated with increased risk of SNHL. ${ }^{8}$ Although these findings suggest that it may be possible to identify children with asymptomatic congenital CMV infection at risk for SNHL in whom monitoring should be considered, additional studies with larger sample sizes and longer audiologic follow-up are needed to assess the relationship between brain imaging abnormalities and SNHL in asymptomatic congenital CMV infection. 
Delayed-onset, progressive and fluctuating SNHL in children with congenital CMV infection has been described in several studies. ${ }^{9}$ In the largest follow-up study describing SNHL and hearing threshold variability in children with congenital CMV infection, the median age at the last audiological evaluation of all children was 5 years. ${ }^{10}$ In the present study, we compared hearing trajectories among children with symptomatic and asymptomatic congenital CMV infection identified with SNHL from infancy through age 18 years and assessed brain abnormalities associated with SNHL among children with asymptomatic congenital CMV infection.

\section{METHODS}

Our study included infants with congenital CMV infection enrolled in the Congenital CMV Longitudinal Study $6,8,11$, identified by routine CMV screening of all newborns at Women's Hospital of Texas, Houston TX, during 1982-1992, or through referrals during 1983-2005. ${ }^{7}$ Congenital CMV infection was diagnosed by culture of urine collected within 3 days (routine screening) to 3 weeks of life (referrals).

We classified congenitally infected infants as symptomatic or asymptomatic case-patients based on the presence or absence, respectively, of at least one of the following CMV-related signs at birth: purpura/petechiae, jaundice, hepatosplenomegaly, microcephaly, unexplained neurological abnormality, elevated liver enzymes (alanine aminotransferase >100 IU), hyperbilirubinemia (total bilirubin $>3 \mathrm{mg} / \mathrm{dl}$ ), hemolytic anemia, or thrombocytopenia (platelet count $<75000 / \mathrm{mm}^{3}$ ). Infants who were small for gestational age (SGA) or had congenital SNHL in the absence of at least one of the above signs were classified as asymptomatic case-patients. ${ }^{12}$ The Institutional Review Board for Human Subject Research for Baylor College of Medicine and Affiliated Hospitals approved the study protocol.

Case-patients had neuroimaging evaluations performed by unenhanced head CT scans by 8 months of age and received hearing evaluations (tympanometry, auditory brainstem response (ABR) and behavioral audiometry) annually up to age 6 years and at least once during elementary, middle and high school years. As per study protocol, during the neonatal period, case-patients were screened using broadband click (2-4 kHz) ABR at $35 \mathrm{~dB}$. At age $0-2$ years (or older for case-patients with cognitive impairment), ABR was performed under conscious sedation using either broadband click or frequency-specific tone burst stimuli $(0.5$ to $8 \mathrm{kHz}$ ). At age $\geq 3$ years, behavioral audiometry was performed using pure tone air and bone conduction testing ( 0.25 to $8 \mathrm{kHz})$. Currently, Texas Children's Hospital adheres to a 2-step newborn hearing screening protocol using otoacoustic emissions, and for those who fail, automated ABR.

For this analysis, we combined ABR with pure-tone air conduction thresholds as follows: 1) ABR response threshold elicited by broadband click stimuli with pure-tone thresholds at 2 and $4 \mathrm{kHz}$, and 2) frequency-specific ABR response threshold elicited by tone-burst stimuli with pure-tone thresholds, after subtraction of $10 \mathrm{~dB}$ for $0.5,1$ and $8 \mathrm{kHz}$, and $0 \mathrm{~dB}$ for 2 and $4 \mathrm{kHz}$ from the tone-burst thresholds. ${ }^{13}$ We excluded assessments in which tympanometry suggested presence of conductive hearing loss. For any audiometric frequency with 'no response' recorded at the maximum output of the hearing testing 
equipment, we estimated the hearing threshold by adding $5 \mathrm{~dB}$ to the maximum output of the equipment. We defined SNHL as $\geq 25 \mathrm{~dB}$ hearing threshold for the broadband click ABR or at any frequency for the corrected tone-burst or pure-tone air conduction results. We categorized SNHL for each ear as congenital/early-onset when detected by the first ABR assessment at age $\leq 2$ months and confirmed in subsequent assessments, or as delayed-onset when detected after one or more assessments with normal hearing, as previously described. ${ }^{6}$ We categorized frequency-specific hearing thresholds as normal when $\unlhd 5 \mathrm{~dB}$, and the severity of hearing loss as follows: slight (16-25 dB), mild (26-40 dB), moderate (41-55 $\mathrm{dB})$, moderately severe $(56-70 \mathrm{~dB})$, severe $(71-90 \mathrm{~dB})$, and profound $(>90 \mathrm{~dB}) .{ }^{14}$

To compare hearing trajectories, i.e. hearing thresholds at each audiometric frequency from 0.5 to $8 \mathrm{kHz}$ for each ear with increasing age, among symptomatic and asymptomatic casepatients, we used growth curve modeling. This is a multilevel regression technique used for longitudinal data to simultaneously analyze both group-level effects and individual-level effects. ${ }^{15}$ As an exploratory step, we first plotted the observed data using smoothers (i.e. splines), overlaying the individual profiles with the average trend lines at each hearing level. We subsequently plotted the corresponding fixed effects models from ordinary least squares regression, i.e. the predicted mean stratified by hearing status and status at birth. We fit models that included the child's age, status at birth (i.e., symptomatic vs. asymptomatic), hearing status (congenital/early-onset SNHL, delayed-onset SNHL, or normal hearing), and an interaction term for age and hearing status. We did not adjust for multiple comparisons because the number of variables included in the models was small. We used SAS's MIXED procedure, incorporating random effects for intercepts (starting hearing thresholds) and slopes (rate of change in hearing thresholds) and a variance component covariance structure.

To assess whether selected abnormal head CT findings of tissue destruction (i.e. intracranial calcification, white matter lucency) within 8 months of birth were associated with SNHL among asymptomatic case-patients, we used the Cox proportional hazards regression model and the Firth method to reduce small sample size bias. ${ }^{16}$ We calculated hazard ratios (HR) and $95 \%$ confidence intervals $(\mathrm{CI})$, and considered results with a P-value $<0.05$ as statistically significant. For all analyses, we used SAS version 9.3 (SAS Institute Inc., Cary, NC, USA).

\section{RESULTS}

Our study included 168 infants with congenital CMV infection: 96 (57\%) case-patients identified through routine CMV screening during 1982-1992, including 4 (4\%) symptomatic and 92 asymptomatic case-patients; and 72 symptomatic case-patients identified through referrals, among whom 51 (71\%) were born before and 21 (29\%) after the Texas Newborn Hearing Screening Program was established in 1999.

Among 76 symptomatic case-patients, 44 (58\%) had congenital/early-onset SNHL: 26 (59\%) had evaluations by both ABR and behavioral audiometry, and 18 (41\%) only by ABR. Among 92 asymptomatic case-patients, 9 (10\%) had congenital/early-onset SNHL, all of whom had evaluations both by ABR and behavioral audiometry. Eight (10\%) of 83 asymptomatic case-patients without congenital/early-onset SNHL were not further evaluated 
by ABR. The median ages at the first and last ABR and behavioral audiometry for symptomatic and asymptomatic case-patients, with or without congenital/early-onset SNHL, are shown in Table 1.

At the end of follow-up, 56 (74\%) symptomatic case-patients and 20 (22\%) asymptomatic case-patients had SNHL (Figure 1), among whom 47 (84\%) and 10 (50\%) had bilateral SNHL, respectively. The majority (72\%) of the 47 symptomatic case-patients with bilateral loss had congenital/early-onset bilateral loss compared with $10 \%$ of the asymptomatic casepatients $(\mathrm{P}<0.001)$.

Our analysis using growth curve modeling to assess hearing trajectories included both symptomatic and asymptomatic case-patients, among whom $78(51 \%)$ and $10(5 \%)$ ears were diagnosed with congenital/early-onset SNHL, respectively, $25(17 \%)$ and $20(11 \%)$ with delayed-onset SNHL, 49 (32\%) and 154 (84\%) ears with normal hearing. Frequencyspecific hearing thresholds were significantly worse in ears with congenital/early-onset SNHL compared to ears with delayed-onset SNHL, especially after 2-3 years of age (Figure 2). We found significant differences in frequency-specific hearing thresholds between symptomatic and asymptomatic case-patients only at 0.5 and $1 \mathrm{kHz}$, but not at any of the higher frequencies (Table 2).

SNHL severity worsened with age for both symptomatic and asymptomatic case-patients. Severity worsened for both congenital/early-onset and delayed-onset SNHL, and at all frequency-specific hearing thresholds (Table 2). Both among symptomatic and asymptomatic case-patients, ears with congenital/early-onset SNHL progressed to severe/ profound loss in all frequencies. Ears with congenital/early-onset SNHL had mean frequency-specific hearing thresholds in the range of moderate to severe at birth, progressing to severe/profound loss by early childhood (Figure 2). Ears with delayed-onset SNHL had mean frequency-specific hearing thresholds in the range of moderate to moderately severe later in childhood (Figure 2). Overall, frequency-specific hearing thresholds deteriorated at a rate of 1 to $3 \mathrm{~dB}$ per year for ears with congenital/early-onset SNHL and 1 to $4 \mathrm{~dB}$ per year for ears with delayed-onset SNHL (Table 2).

Among 92 asymptomatic case-patients, 87 (95\%) had a head CT scan performed within the first 8 months of life. Eighteen (20\%) asymptomatic case-patients had abnormal findings categorized as tissue destruction: $11(61 \%)$ had white matter lucency, $5(28 \%)$ had intracranial calcifications, and $2(11 \%)$ had malacia. Head CT scans of asymptomatic casepatients showing white matter lucency (periventricular leukomalacia) not associated with prematurity and punctate periventricular punctate calcifications are shown in Figure 3.

The proportion of asymptomatic case-patients with congenital/early-onset SNHL was greater among those with brain abnormalities than those without, but the associations were not statistically significant (Table 3). By age 5 years, SNHL was diagnosed in 4/11 (36\%) asymptomatic case-patients with vs. $6 / 67$ (9\%) without white matter lucency (HR=4.4; 95\% CI: $1.3-15.6 ; \mathrm{P}<0.05)$. SNHL by age 18 years was also significantly associated with white matter lucency (Table 3). 


\section{DISCUSSION}

In this study based on longitudinal hearing assessments from infancy up to 18 years of age, congenital/early-onset SNHL frequently progressed to severe or profound hearing loss by 5 years of age, regardless the presence of CMV-related signs at birth. A majority of the asymptomatic case-patients with congenital/early-onset SNHL had unilateral loss at onset but most of these children developed SNHL in the contra-lateral ear by age 18 years. The progression of delayed-onset SNHL was similar among asymptomatic and symptomatic case-patients. Children with congenital/early-onset SNHL had worse hearing thresholds by 18 years of age on average when compared with children with delayed-onset SNHL.

Universal newborn screening for congenital CMV infection has the potential to identify children at risk for SNHL who could potentially benefit from regular monitoring and earlier intervention. ${ }^{17} \mathrm{~A}$ better understanding of age of onset and risk factors for SNHL in children with asymptomatic congenital CMV infection could inform clinical guidance regarding frequency, duration and type of audiological monitoring these children should receive. A recent study suggested that either universal or targeted newborn screening, a strategy of CMV testing of infants who failed the newborn hearing screening, appear to be costeffective. ${ }^{18}$ However, no additional cost was assumed for public health infrastructure required for CMV screening using saliva specimens. ${ }^{19,20}$ Additionally, the study assumed $1 \%$ of infants with asymptomatic congenital CMV infection would require further neuroimaging evaluation by magnetic resonance imaging (MRI). ${ }^{18}$ In contrast, $20 \%$ of the asymptomatic case-patients in our cohort had brain imaging abnormalities. More data is needed to inform the evaluation of the potential costs and benefits of newborn screening for CMV.

We found that children with asymptomatic congenital CMV infection who had white matter lucency were roughly 4 times as likely to be diagnosed with SNHL by age 5 years as those without white matter lucency. In another study, white matter lucency was associated with a retrospective diagnosis of asymptomatic congenital CMV infection in children who later developed static encephalopathy. ${ }^{21}$ To avoid ionizing radiation from CT, cranial ultrasound and MRI are currently the preferred methods for neuroimaging evaluation of infants with congenital CMV infection. ${ }^{22}$ Although cranial ultrasound is a relatively low cost technique that is readily available in most centers, it does not reveal white matter abnormalities. ${ }^{22}$ MRI is recommended for investigation of cortical, white matter and cerebellar lesions in congenital CMV infection but requires professional expertise and may not be widely available. ${ }^{23}$ Consensus guidance on using neuroimaging to evaluate infants with asymptomatic congenital CMV infection is needed. Furthermore, the definition of symptomatic congenital CMV disease is likely to evolve as prognostic markers of long-term impairments are elucidated and data from clinical trials of antiviral treatment of newborns with asymptomatic congenital CMV infection and isolated SNHL become available. ${ }^{24}$ Currently, antivirals are not recommended for routine use in this population.

The development and maturation of the auditory system occurs in a stepwise fashion. The cochlea acquires an adult-like configuration by the end of the second trimester of pregnancy, but the myelination continues throughout the perinatal period, early and later childhood. ${ }^{25}$ 
Previous neuroimaging studies of infants with congenital CMV infection have suggested that the pattern of brain abnormalities varies with timing of CMV infection during pregnancy. ${ }^{26}$ Loss of neurons and glia suggest CMV infection before 18 weeks of pregnancy. Migrational abnormalities, such as polymicrogyria, which have been observed in symptomatic but not in asymptomatic case-patients, suggest CMV infection during 18-24 weeks gestation. Delayed myelination, dysmyelination, and white matter disease (as observed in our asymptomatic case-patients), suggest CMV infection at $>26$ weeks of pregnancy. ${ }^{26}$ It is possible that CMVrelated SNHL could occur as a consequence of fetal infection at any time during pregnancy. A better understanding of brain imaging findings associated with CMV-related SNHL could provide insights into SNHL pathogenesis and timing of CMV infection during pregnancy. Animal models have suggested that the potential mechanisms for CMV-related SNHL are host derived inflammatory responses and not direct virus-mediated cytopathology. ${ }^{27} \mathrm{~A}$ better understanding of the mechanisms for CMV-related SNHL could inform development of therapeutic options to prevent onset and further progression of SNHL in children with congenital CMV infection. ${ }^{28}$

Our study had limitations. Symptomatic case-patients were identified through referrals and likely reflect relatively severe cases. ${ }^{29}$ The small number of asymptomatic case-patients with congenital/early-onset SNHL limited the statistical power to find significant associations with brain abnormalities and congenital/early-onset SNHL. Different audiologic equipment were used over the years and the variation in maximum intensity output at each frequency over the course of the study could have resulted in underestimates of hearing thresholds for some frequencies. Data were not available for all audiometric frequencies at all ages because testing of many symptomatic case-patients with developmental delays or cognitive impairment relied on ABR. Frequency-specific tone-burst ABR was not available for all case-patients at younger ages, which could have resulted in missed SNHL.

In this study, we used growth curve modeling to analyze longitudinal data on hearing thresholds. Growth curve modeling includes both fixed and random effects to describe the general trajectory for both the group as a whole and for the individuals within the group. ${ }^{30}$ We compared ears with congenital/early-onset SNHL among symptomatic and asymptomatic case-patients and found no differences in hearing thresholds at higher frequencies (2, 4 and $8 \mathrm{kHz})$. The observed means of the hearing thresholds suggested a steeper progression of SNHL during early childhood although the predicted means increased linearly with age. The statistically significant difference in the predicted means of hearing thresholds at the lower frequencies needs further clarification. Many symptomatic casepatients evaluated only by ABR missed data for 0.5 and $1 \mathrm{kHz}$. The differences of 7-15 dB among symptomatic relative to asymptomatic case-patients at 0.5 and $1 \mathrm{kHz}$ may not be clinically relevant considering hearing thresholds in ears with congenital/early-onset SNHL differed from normal hearing ears by $60-85 \mathrm{~dB}$.

Previously, we reported that $5 \%$ of our asymptomatic case-patients identified through newborn screening had SNHL $\geq 70 \mathrm{~dB}$ HL in at least one ear by age 12 months. ${ }^{6}$ This figure corresponds to 900 children annually in the United States, half of whom would meet current candidacy criteria for cochlear implantation by age 5 years. ${ }^{6}$ The CMV and Hearing Multicenter Screening Study, which screened >100,000 newborns for CMV in the United 
States, found a 7-fold rate of failure in newborn hearing screening among congenitally infected compared to uninfected infants. ${ }^{4}$ Nonetheless, in the absence of universal screening for congenital CMV infection, nearly half of the infants with asymptomatic congenital CMV infection who present with SNHL within 8 weeks of life would be missed by newborn hearing screening. ${ }^{4}$ Targeted CMV screening of newborns who fail the newborn hearing screening ${ }^{31}$ will still miss a large portion of infants with congenital CMV infection either symptomatic or asymptomatic at birth who are at risk of developing SNHL early in life. ${ }^{4,20}$

In our cohort, infants with asymptomatic congenital CMV infection who were diagnosed with SNHL during the first 2 years of life had lower full-scale intelligence and receptive vocabulary scores compared to uninfected children. ${ }^{32}$ Although the Joint Committee on Infant Hearing in 2007 endorsed audiologic monitoring of children identified with congenital CMV infection ${ }^{33}$, a consensus including how often, for how long, or by whom testing should be performed is needed. ${ }^{20}$ Infants with congenital CMV infection, regardless the presence of other signs at birth, who pass newborn hearing screening often develop SNHL which can progress to severe or profound levels in early childhood. Therefore, early detection of CMV-related SNHL during infancy is important to allow for timely intervention to optimize language development.

\title{
Acknowledgments
}

We thank all the children who participated in the study, their families and physicians for their lifetime of dedication and support for this study. We also thank Mike Cannon for his role in initiating the CDC cooperative agreement with Baylor College of Medicine.

The Congenital CMV Longitudinal Study Group through the years has included: Shahzad Ahmed, Hanna Baer, MD, Amit R. Bhatt, MD, Peggy Blum, AuD and Texas Children's Hospital Audiology, Frank Brown, MD, Francis Catlin, MD, Alison C. Caviness, MD, PhD, MPH, David K. Coats, MD, Jane C. Edmonds, MD, Marily Flores, MS, Daniel Franklin, MD, Cindy Gandaria, Jewel Greer, Carol Griesser, RN, Mohamed A. Hussein, MD, Isabella Iovino, PhD, Allison Istas, MPH, Haoxing (Douglas) Jin, Mary K. Kelinske, OD, Joseph T. Klingen, Antone Laurente, PhD, Thomas Littman, PhD, Mary Murphy, MS, Jerry Miller, PhD, Christopher Nelson, MD, Daniel Noyola, MD, Evelyn A. Paysse, MD, Alan Percy, MD, Sara Reis, RN, Ann Reynolds, MD, Judith Rozelle, MS, O’Brien Smith, PhD, Paul Steinkuller, MD, Marie Turcich, MS, Sherry Sellers Vinson, MD, Robert G. Voigt, MD, Bethann Walmus, Jill Williams, MA, Daniel Williamson, MD, Kimberly G. Yen, MD, Martha D. Yow, MD, and Gail J. Demmler-Harrison MD.

Funding Sources: The study was supported, in part, by the CMV Research Fund Donors at Baylor College of Medicine; the Woman's Hospital of Texas Research Foundation; the Office of Research Resources and the General Clinical Research Center for Children at Texas Children's Hospital and Baylor College of Medicine (NIH 5M0I RR00188-33); the Mental Retardation Research Center at Baylor College of Medicine (NIH-CHHD 5 P30 HD24064P); the Research to Prevent Blindness, Inc. New York, NY; the Deafness Foundation, Houston, TX; the Vale Ashe Foundation, Houston, TX; the Maddie's Mission Foundation, Katy, TX; the Naymola Foundation, Beaumont, TX; the American Pediatric Society-Society for Pediatric Research Summer Student Research Program (NIH-CHHD); and the Centers for Disease Control and Prevention (Cooperative Agreement FOA IP 10-006).

Dr. Demmler-Harrison's institution received funding from Merck Sharpe \& Dohme Corporation since July 2016 to assist with salary support for further analysis on long term outcomes of congenital CMV infection not included in this report.

\section{Abbreviations}

\author{
ABRh auditory brainstem response \\ CI confidence interval
}




$\begin{array}{ll}\text { CMV } & \text { cytomegalovirus } \\ \text { CT } & \text { computed tomography } \\ \text { HR } & \text { hazard ratio } \\ \text { MRI } & \text { magnetic resonance imaging } \\ \text { SNHL } & \text { sensorineural hearing loss }\end{array}$

\section{References}

1. Grosse SD, Ross DS, Dollard SC. Congenital cytomegalovirus (CMV) infection as a cause of permanent bilateral hearing loss: a quantitative assessment. J Clin Virol. 2008; 41:57-62. [PubMed: 17959414]

2. Boppana SB, Ross SA, Novak Z, et al. Dried blood spot real-time polymerase chain reaction assays to screen newborns for congenital cytomegalovirus infection. JAMA. 2010; 303:1375-82. [PubMed: 20388893]

3. Boppana SB, Ross SA, Shimamura M, et al. Saliva polymerase-chain-reaction assay for cytomegalovirus screening in newborns. N Engl J Med. 2011; 364:2111-8. [PubMed: 21631323]

4. Fowler KB, McCollister FP, Sabo DL, et al. A Targeted Approach for Congenital Cytomegalovirus Screening Within Newborn Hearing Screening. Pediatrics. 2017; 139

5. Rawlinson WD, Boppana SB, Fowler KB, et al. Congenital cytomegalovirus infection in pregnancy and the neonate: consensus recommendations for prevention, diagnosis, and therapy. Lancet Infect Dis. 2017; 17:e177-e88. [PubMed: 28291720]

6. Lanzieri TM, Chung W, Flores M, et al. Hearing Loss in Children With Asymptomatic Congenital Cytomegalovirus Infection. Pediatrics. 2017; 139

7. Lanzieri TM, Leung J, Caviness AC, et al. Long-term outcomes of children with symptomatic congenital cytomegalovirus disease. J Perinatol. 2017; 37:875-80. [PubMed: 28383538]

8. Williamson WD, Demmler GJ, Percy AK, et al. Progressive hearing loss in infants with asymptomatic congenital cytomegalovirus infection. Pediatrics. 1992; 90:862-6. [PubMed: 1331946]

9. Goderis J, De Leenheer E, Smets K, et al. Hearing loss and congenital CMV infection: a systematic review. Pediatrics. 2014; 134:972-82. [PubMed: 25349318]

10. Dahle AJ, Fowler KB, Wright JD, et al. Longitudinal investigation of hearing disorders in children with congenital cytomegalovirus. J Am Acad Audiol. 2000; 11:283-90. [PubMed: 10821506]

11. Yow MD, Williamson DW, Leeds LJ, et al. Epidemiologic characteristics of cytomegalovirus infection in mothers and their infants. Am J Obstet Gynecol. 1988; 158:1189-95. [PubMed: 2835906]

12. Cannon MJ, Griffiths PD, Aston V, et al. Universal newborn screening for congenital CMV infection: what is the evidence of potential benefit? Rev Med Virol. 2014; 24:291-307. [PubMed: 24760655]

13. Stapells DR, Gravel JS, Martin BA. Thresholds for auditory brain stem responses to tones in notched noise from infants and young children with normal hearing or sensorineural hearing loss. Ear Hear. 1995; 16:361-71. [PubMed: 8549892]

14. American Speech-Language-Hearing Association. [Accessed April 26, 2016] Degree of Hearing Loss. Available at: http://www.asha.org/public/hearing/Degree-of-Hearing-Loss/

15. Raudenbush, SW., Bryk, AS. Hierarchical Linear Models: Applications and Data Analysis Methods 2002. 2. Thousand Oaks, CA: Taylor \& Francis; 2002.

16. King G, Zeng L. Logistic Regression in Rare Events Data. Political Analysis. 2001; 9:137-63.

17. Demmler GJ. Screening for congenital cytomegalovirus infection: a tapestry of controversies. J Pediatr. 2005; 146:162-4. [PubMed: 15689898] 
18. Gantt S, Dionne F, Kozak FK, et al. Cost-effectiveness of Universal and Targeted Newborn Screening for Congenital Cytomegalovirus Infection. JAMA Pediatr. 2016; 170:1173-80. [PubMed: 27723885]

19. Grosse SD, Dollard S, Ross DS, et al. Newborn screening for congenital cytomegalovirus: Options for hospital-based and public health programs. J Clin Virol. 2009; 46(Suppl 4):S32-6. [PubMed: 19783205]

20. Grosse SD, Dollard SC, Kimberlin DW. Screening for Congenital Cytomegalovirus After Newborn Hearing Screening: What Comes Next? Pediatrics. 2017; 139

21. van der Knaap MS, Vermeulen G, Barkhof F, et al. Pattern of white matter abnormalities at MR imaging: use of polymerase chain reaction testing of Guthrie cards to link pattern with congenital cytomegalovirus infection. Radiology. 2004; 230:529-36. [PubMed: 14752192]

22. Capretti MG, Lanari M, Tani G, et al. Role of cerebral ultrasound and magnetic resonance imaging in newborns with congenital cytomegalovirus infection. Brain Dev. 2014; 36:203-11. [PubMed: 23647916]

23. Lanari M, Capretti MG, Lazzarotto T, et al. Neuroimaging in CMV congenital infected neonates: how and when. Early Hum Dev. 2012; 88(Suppl 2):S3-5.

24. Park, A. [Accessed August, 8, 2017] Randomized Controlled Trial of Valganciclovir for Asymptomatic Cytomegalovirus Infected Hearing Impaired Infants (ValEAR). 2017. Available at: https://clinicaltrials.gov/ct2/show/NCT03107871

25. Moore JK, Linthicum FH Jr. The human auditory system: a timeline of development. Int J Audiol. 2007; 46:460-78. [PubMed: 17828663]

26. Barkovich AJ, Lindan CE. Congenital cytomegalovirus infection of the brain: imaging analysis and embryologic considerations. AJNR Am J Neuroradiol. 1994; 15:703-15. [PubMed: 8010273]

27. Bradford RD, Yoo YG, Golemac M, et al. Murine CMV-induced hearing loss is associated with inner ear inflammation and loss of spiral ganglia neurons. PLoS Pathog. 2015; 11 :e1004774. [PubMed: 25875183]

28. Sidell D, Ward JA, Pordal A, et al. Combination therapies using an intratympanic polymer gel delivery system in the guinea pig animal model: A safety study. Int J Pediatr Otorhinolaryngol. 2016; 84:132-6. [PubMed: 27063768]

29. Dollard SC, Grosse SD, Ross DS. New estimates of the prevalence of neurological and sensory sequelae and mortality associated with congenital cytomegalovirus infection. Rev Med Virol. 2007; 17:355-63. [PubMed: 17542052]

30. Curran PJ, Obeidat K, Losardo D. Twelve Frequently Asked Questions About Growth Curve Modeling. J Cogn Dev. 2010; 11:121-36. [PubMed: 21743795]

31. Diener ML, Zick CD, McVicar SB, et al. Outcomes From a Hearing-Targeted Cytomegalovirus Screening Program. Pediatrics. 2017; 139

32. Lopez AS, Lanzieri TM, Claussen AH, et al. Intelligence and Academic Achievement With Asymptomatic Congenital Cytomegalovirus Infection. Pediatrics. 2017; 140

33. American Academy of Pediatrics, Joint Committee on Infant Hearing. Year 2007 position statement: Principles and guidelines for early hearing detection and intervention programs. Pediatrics. 2007; 120:898-921. [PubMed: 17908777] 


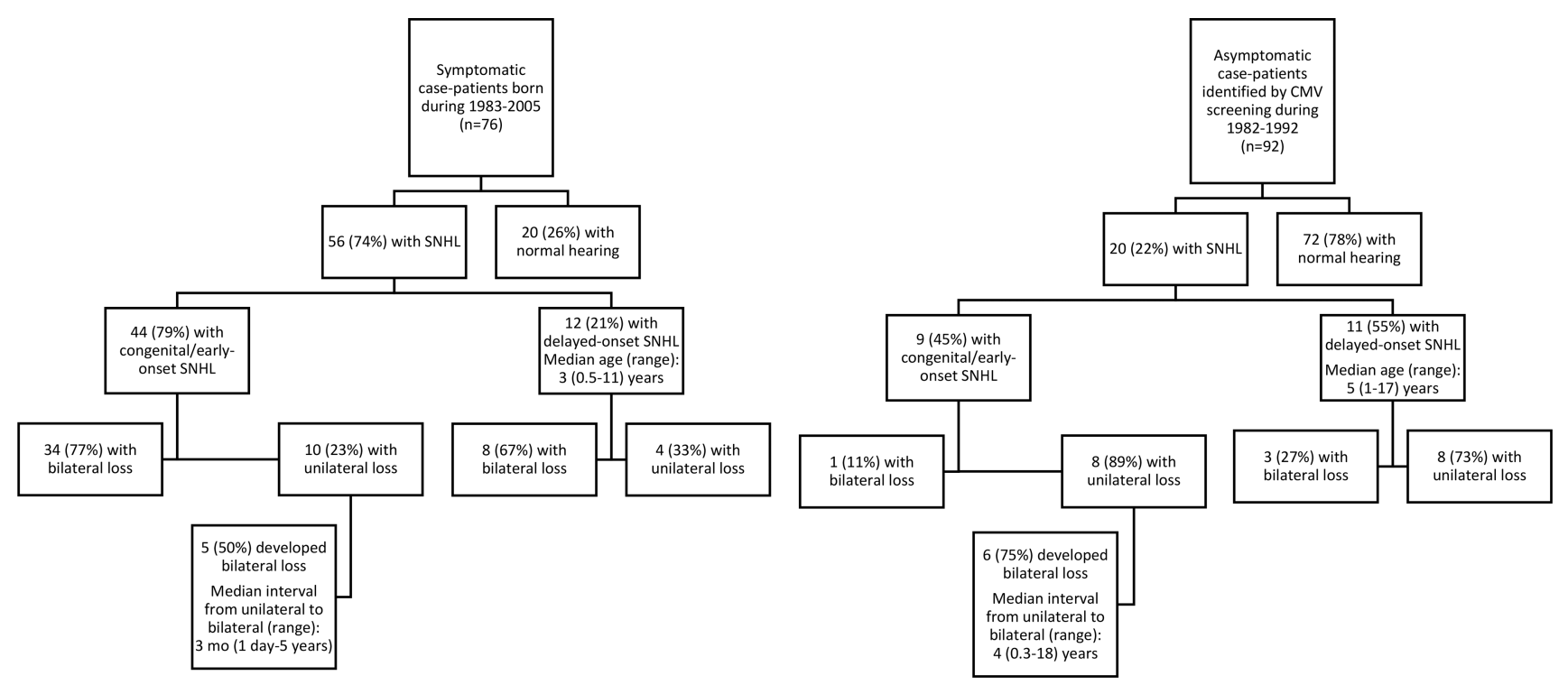

Figure 1.

Congenital CMV Longitudinal Study Cohort. 
Frequency

$0.5 \mathrm{kHz}$

$1 \mathrm{kHz}$
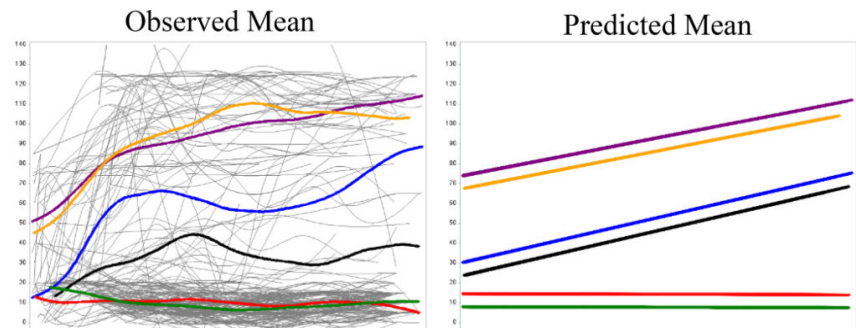

$2 \mathrm{kHz}$

$4 \mathrm{kHz}$

$8 \mathrm{kHz}$
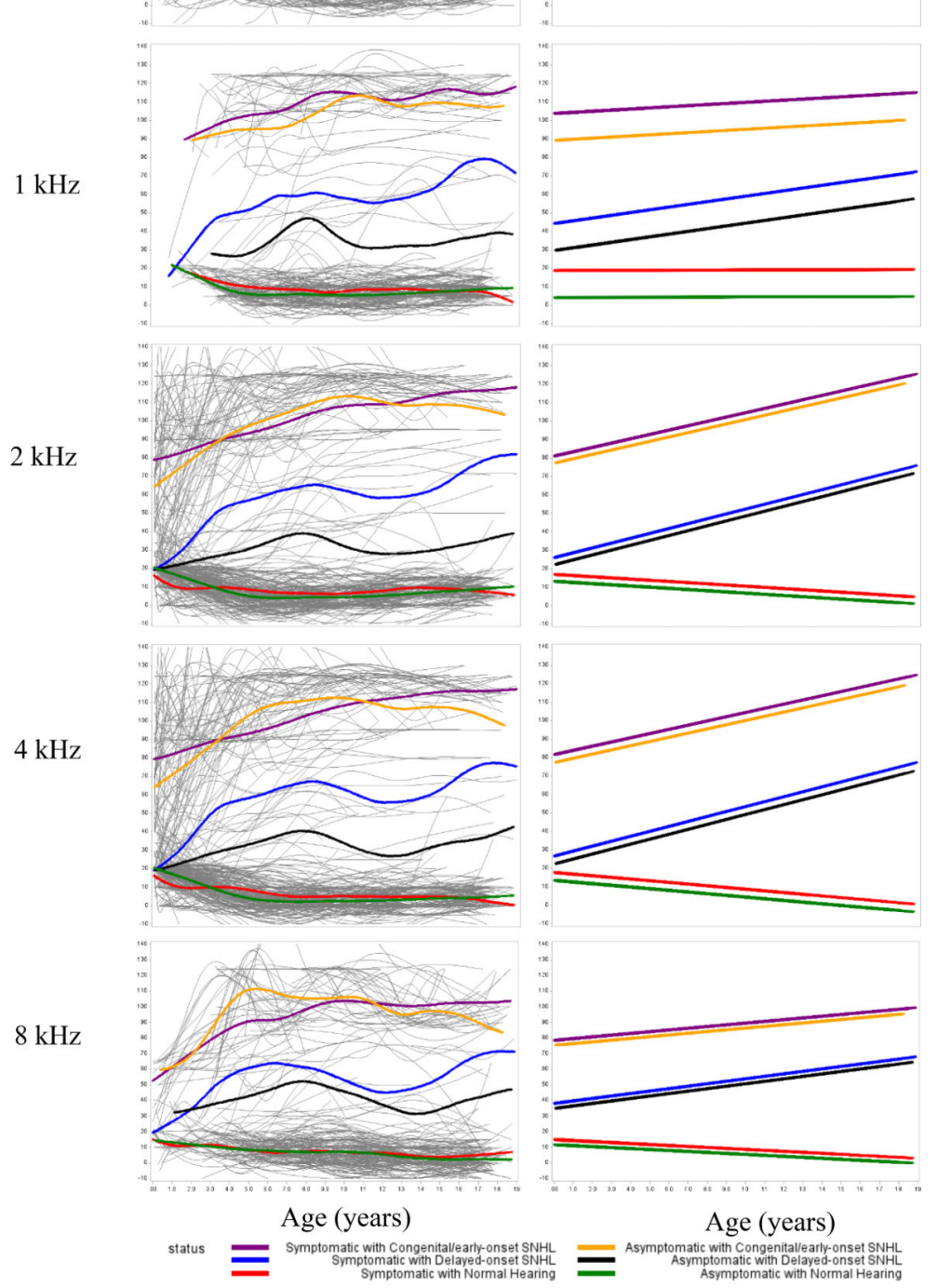

Age (years)

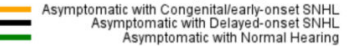

Figure 2.

Frequency-specific hearing trajectories by age for ears with congenital/early-onset SNHL, delayed-onset SNHL, and normal hearing, among symptomatic and asymptomatic casepatients.

Caption: The "spaghetti plots" in the left-hand column fit the raw data using smoothers (i.e. splines). Individual profiles are overlaid with the average trend lines. The plots in the righthand column show the predicted means of hearing thresholds using the corresponding fixed effects models from ordinary least squares regression. 

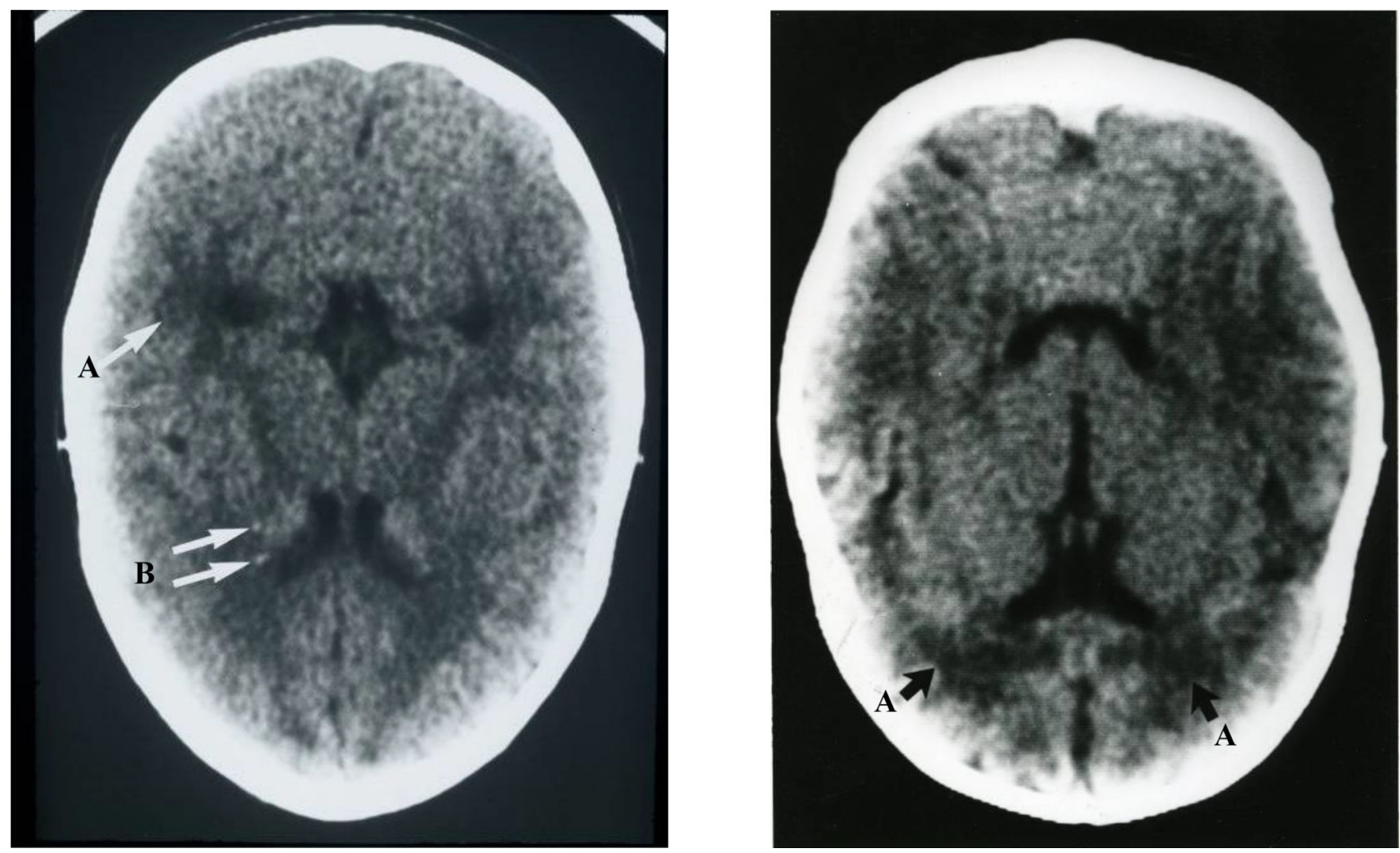

Figure 3.

Head CT scans from children with asymptomatic congenital CMV infection.

Caption: A - white matter lucency (periventricular leukomalacia) not associated with prematurity. B -periventricular punctate calcification. 


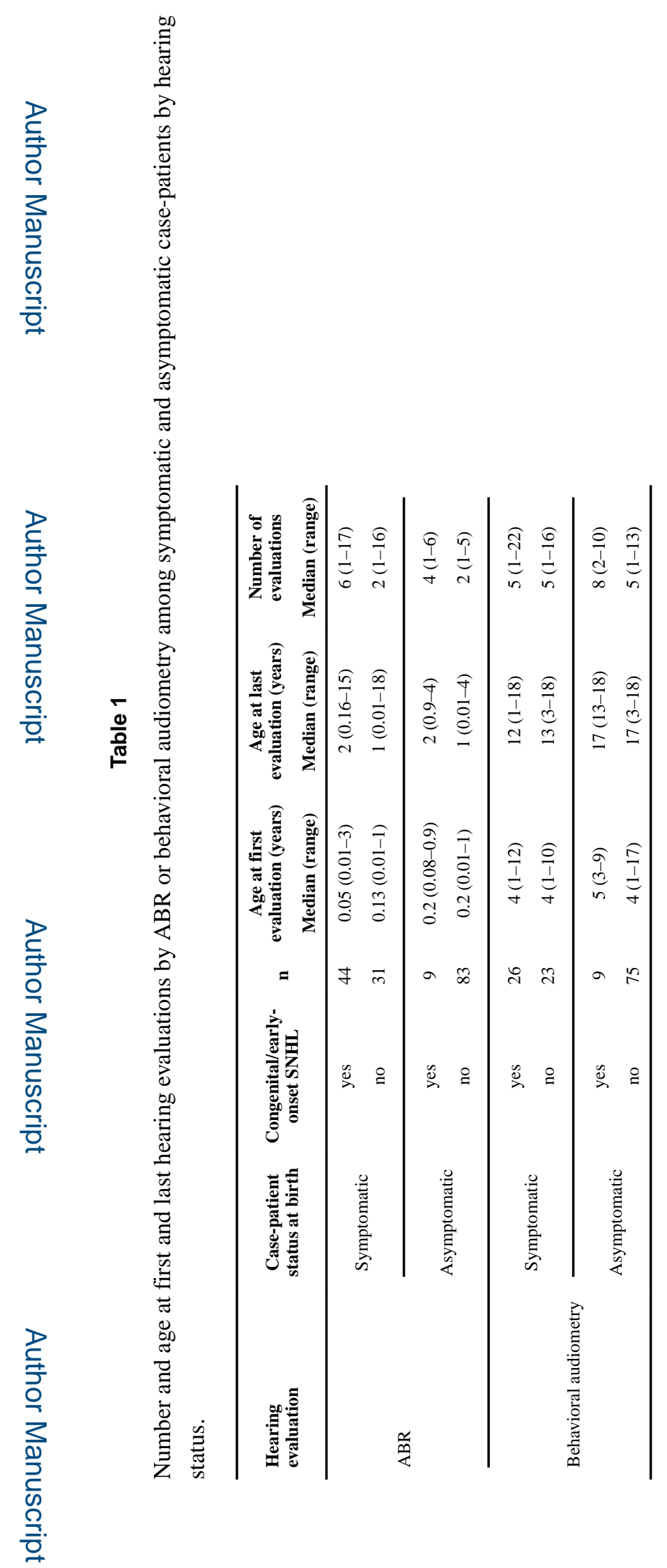

Otolaryngol Head Neck Surg. Author manuscript; available in PMC 2018 October 01. 


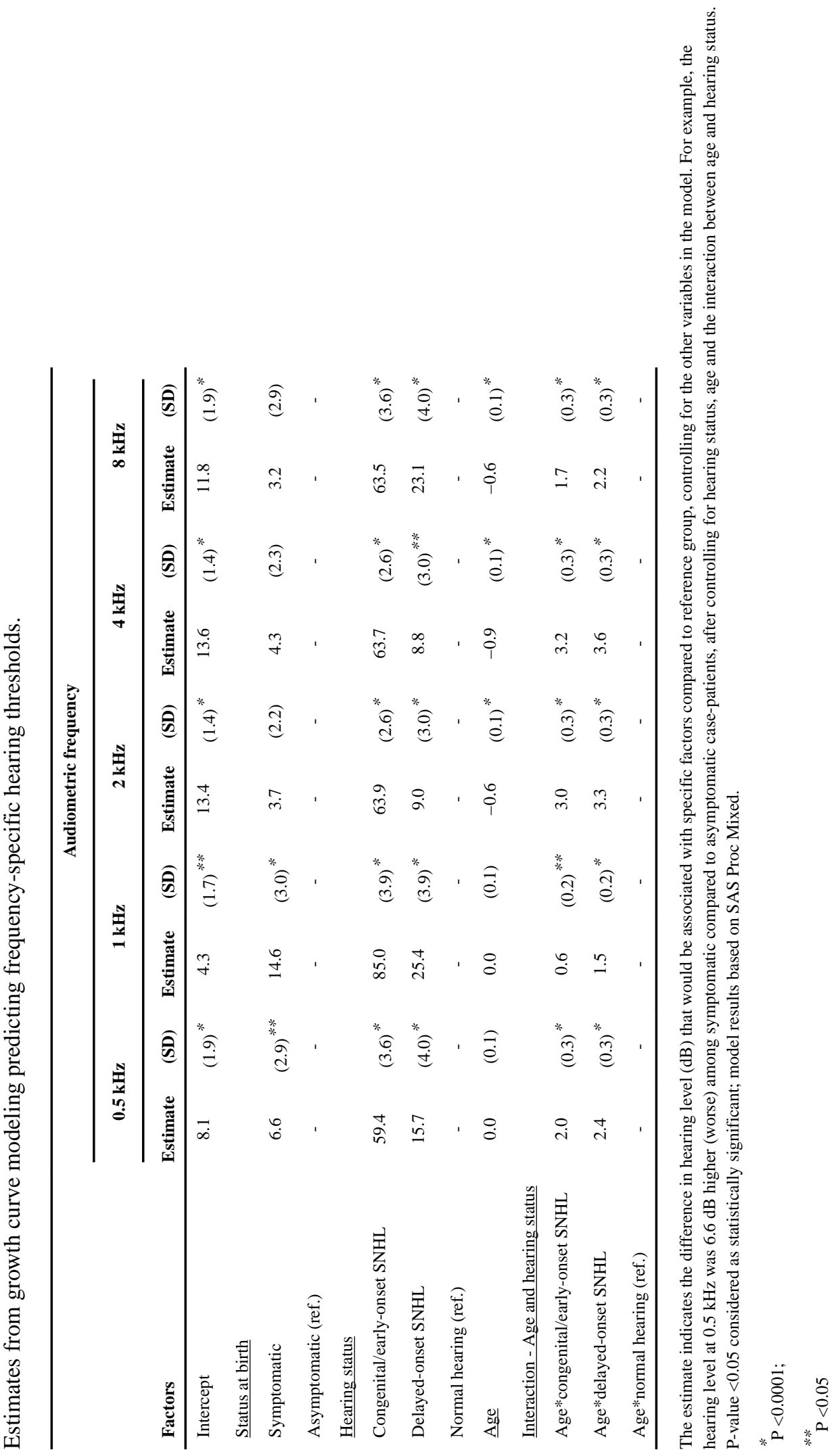

Otolaryngol Head Neck Surg. Author manuscript; available in PMC 2018 October 01. 


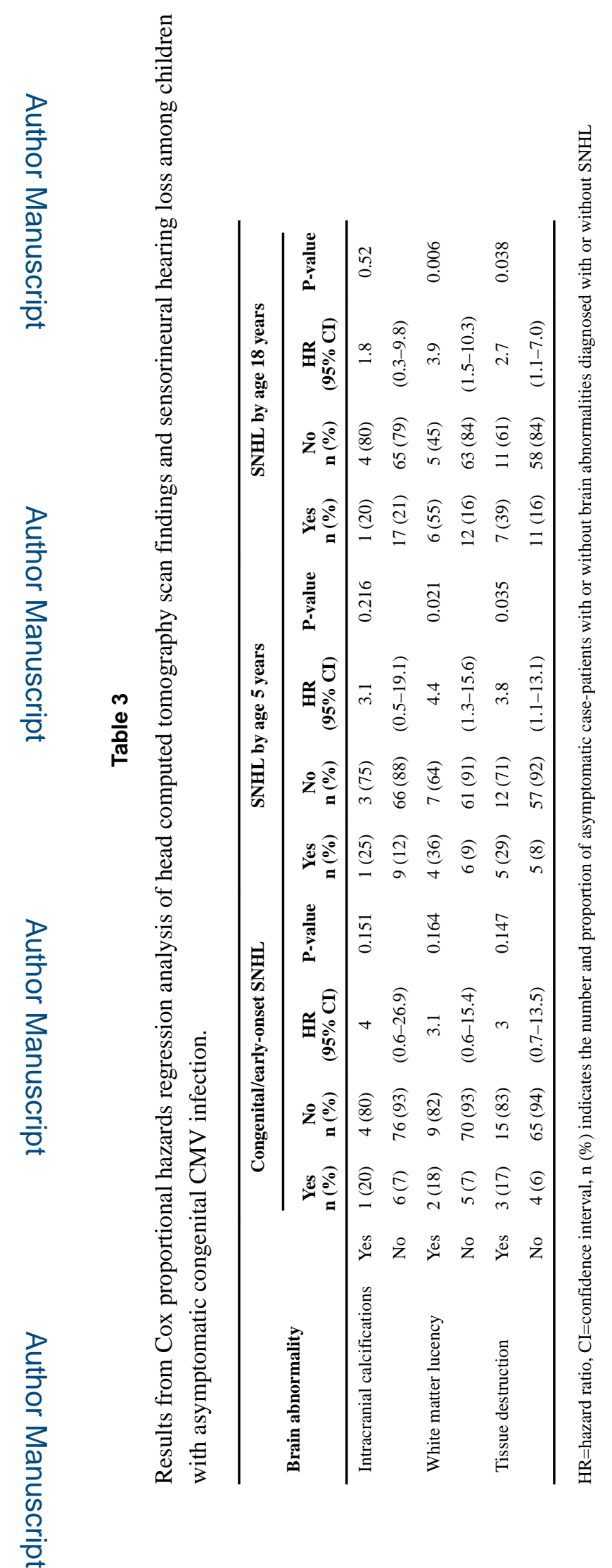

Otolaryngol Head Neck Surg. Author manuscript; available in PMC 2018 October 01. 